\title{
Speeding-up Network Reconfiguration by Minimum Cost Maximum Flow Based Branch Exchanges
}

\author{
Cristinel Ababei Member, IEEE, Rajesh Kavasseri, Member, IEEE
}

\begin{abstract}
We propose a novel and efficient heuristic algorithm for solving the distribution network reconfiguration problem for loss reduction. We formulate the problem of finding incremental branch exchanges as a minimum cost maximum flow (MCMF) problem. This novel approach finds the best set of concurrent branch exchanges during each iteration of the algorithm and leads to larger loss reductions and a reduced number of iterations, hence significantly reducing the computational runtime. Experiments using distribution systems with sizes of up to 10476 buses demonstrate that the proposed technique leads to an average speed-up of $2.3 \times$ with similar or better solution quality compared to the Baran's reconfiguration technique [32].
\end{abstract} time.

Index Terms-Power systems, network reconfiguration, run-

\section{INTRODUCTION}

$\mathbf{N}$ ETWORK reconfiguration of power distribution systems is defined as the change in the network structure as a result of closing tie and opening sectionalizing switches. Its main goals are to ensure service restoration under contingencies, to reduce losses, and to balance load in the system, under the constraint of maintaining the radiality of the network. Network reconfiguration has been identified as a primary mechanism that has a direct impact on reliability, efficient service restoration and maintenance of optimal operating conditions. For example, Con Edison has proposed a third generation (G3) distribution network whose features include flexible reconfiguration, super-fast simulators, advanced visualization tools, and adaptive response systems [1]. Such features are indispensable in fulfilling the vision of a self healing grid that can automatically respond to disturbances while continuously optimizing the overall performance. Electric Power Research Institute (EPRI)'s own endeavors to develop D-FSM (Distribution Fast Simulation and Modeling) [2] confirm the importance and need to develop a super-fast computational platform that can provide in real time information necessary to facilitate several distribution automation (DA) functions and system level lookahead capabilities.

Network reconfiguration is a combinatorial problem and one needs to compute the power flow solution for every topological change resulting due to reconfiguration. Hence, the efficiency of the reconfiguration algorithm depends on both the efficiency of the power flow solution technique (which has to be readily available multiple times in order to evaluate loss reductions) and the efficiency of the reconfiguration approach itself.

C. Ababei and R. Kavasseri are with the Department of Electrical and Computer Engineering, North Dakota State University, Fargo ND, 58105, USA (e-mail: cristinel.ababei@ndsu.edu; rajesh.kavaseri@ndsu.edu)
The network reconfiguration problem has been the subject of extensive previous research. Most of the previous work can be divided into three main categories: evolutionary and knowledge based techniques [3]-[27], heuristics [31]-[50], and mixed methods [51]-[55]. Even though evolutionary and knowledge based techniques can handle broader objectives, they suffer from very long computational times and therefore are less suitable for online distribution automation. Examples of such techniques include genetic algorithm (GA) [3],[4], refined GA [5], tabu search [6],[7], ant colony [8], simulated annealing [9],[10], artificial neural network based [11], particle swarm optimization [12],[13], fuzzy mutated GA [14], and fuzzy multi-objective [15].

Hybrid reconfiguration approaches are mixed solutions that combine evolutionary and heuristic techniques in order to shorten the computational time without sacrificing solution quality. Even though their runtimes are generally shorter than of evolutionary techniques, they are still computationally demanding compared to heuristic approaches. Heuristic algorithms for network reconfiguration have been proved to offer excellent results with significantly shorter runtimes. Thus, they are among the best candidates for real time distribution system reconfiguration for loss minimization [28]-[30]. For instance, Civanlar et al. [31] proposed an efficient reconfiguration algorithm based on the idea of branch-exchange for loss reduction. This algorithm was later improved by Baran and $\mathrm{Wu}$ [32], who also proposed an algebraic expression for estimating loss reduction due to branch exchanges.

In this paper, we propose and implement a novel heuristic technique based on the minimum cost maximum flow (MCMF) algorithm to improve the efficiency of reconfiguration while maintaining or improving the quality of the final solution. Similar to previous heuristic approaches, our technique is iterative in nature and uses first-order branch exchanges to reconfigure the network and to minimize losses. The novelty of our technique lies in the network-flow based modeling of the problem of branch exchanges during each iteration. The solution of the network-flow problem is given by the best set of concurrent branch exchanges for maximum loss reduction. By performing concurrent branch exchanges during each iteration, the overall convergence of the algorithm is improved and the runtime is shortened significantly due to the reduced number of iterations. We applied the proposed reconfiguration method to systems with sizes of up to 10476 buses and achieved speed-ups of up to $2.3 \times$ compared to the Baran's reconfiguration technique [32]. 


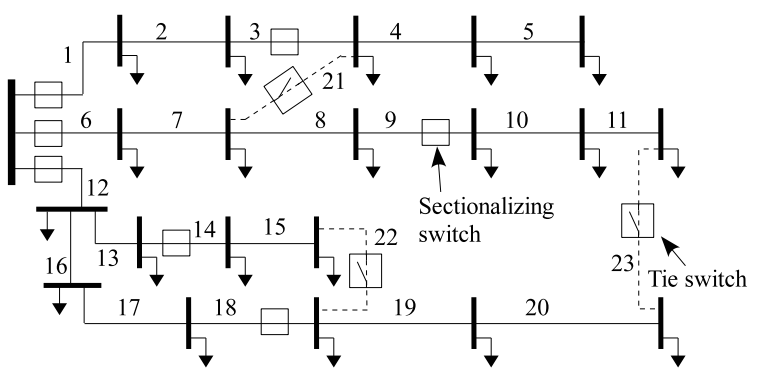

Fig. 1. One line diagram of an example of radial distribution system.

\section{Network Flow Based Reconfiguration}

\section{A. Preliminaries}

Before we introduce the proposed technique we discuss the definition of branch exchange and the power flow solution, using the simple system from Fig. 1. In order to simplify the presentation, we present the system on a per phase basis. The loads of a feeder section are assumed to be constant P,Q loads located at the end of the lines. In Fig. 1, solid branches represent the lines that constitute the current in service base radial configuration. Some of these branches have switches, which are closed and represented by small rectangles. These switches are referred to as sectionalizing switches. The dotted branches $(21,22,23)$ represent the lines with open switches, referred to as tie switches. The network can be reconfigured by closing tie and opening sectionalizing switches. The process of closing a tie switch and opening a sectionalizing switch is referred to as branch exchange. For example, the line 23 can be closed. Because this will create a loop (formed by lines 6,7,8,9,10,11,12,16,17,18,19,20,23), a branch with a sectionalizing switch will have to be open in order to restore the radiality of the system. In this example any of the switches $6,9,12,18$ can be open in order to break the loop. If for example, switch 18 will be open, the loads between branches 18-20 will be transferred from one feeder to the other. Branch exchanges provide an efficient mechanism to perform network reconfiguration in order to address changing operation conditions. Two of the main purposes of network reconfiguration are loss reduction and load balancing. In this paper, our focus is on network reconfiguration for loss reduction.

In order to be able to compute current losses inside the network as well as to estimate the loss change due to a branch exchange, one needs the power flow solution (PFS). In order to compute the power flow solution, the feeder (or substation) voltage is assumed to be constant, lines are represented by series impedances, and loads are assumed constant power sinks. Shunt capacitors are represented as reactive power injections. For the purpose of developing the technique proposed in this paper, we implemented the power flow solution DistFlow reported in [56], which is very popular and has been proved to be very efficient. DistFlow provides also an efficient technique to estimate the loss reduction due to a given branch exchange. Because the focus of this work is on network reconfiguration and not on power flow solution, the reader is encouraged to consult [56] for details of DistFlow

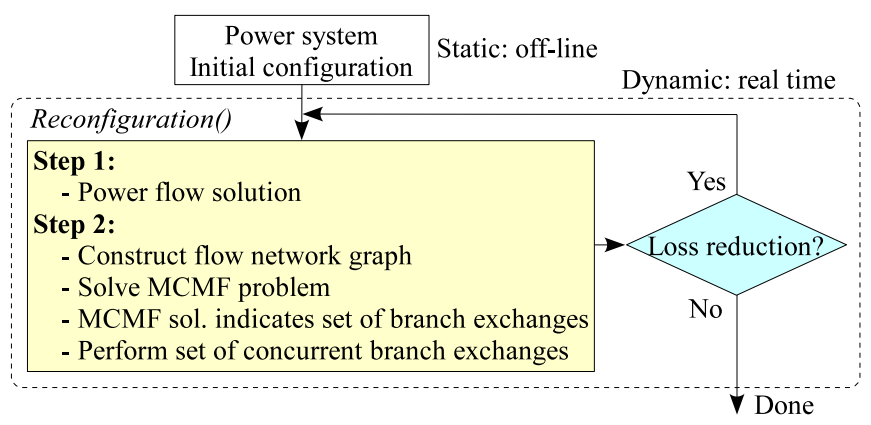

Fig. 2. Diagram of the proposed network-flow based reconfiguration algorithm.

PFS. The distribution power flow solution will be used as a subroutine in each iteration of the proposed reconfiguration technique.

\section{B. New Reconfiguration Algorithm}

The proposed network reconfiguration algorithm is an iterative heuristic algorithm described in Fig. 2. Each main iteration of the algorithm has two main steps. In the first step, the power flow solution for the current configuration is computed using the DistFlow PFS method from [56]. In the second step, we search for incremental network changes (implemented via branch exchanges) that lead to large loss reductions. The novelty of our approach lies in the enhanced branch exchange technique employed for this search. Using a minimum cost maximum flow (MCMF) based modeling approach, we find sets of multiple branch exchanges that are implemented concurrently during each iteration. This is done by constructing and solving the MCMF problem and then identifying from the MCMF solution the set of concurrent branch exchanges; which will be described in detail in the following section. This two-step reconfiguration technique is repeated until no significant improvement between two consecutive iterations is achieved.

The merit of the proposed reconfiguration technique is in the MCMF formulation of the problem of finding concurrent branch exchanges. This approach improves the local optimality of the reconfiguration solution during each iteration of the algorithm and leads to larger loss reductions (during each iteration) and a reduced number of iterations, hence significantly reducing the runtime.

\section{Network Flow Based Multiple Concurrent Branch Ex- changes}

In this section we present the MCMF based modeling that we use to construct the MCMF problem whose solution will indicate the best set of concurrent branch exchanges. For this purpose, we use a simple radial power distribution system example presented in Fig. 3.

The proposed reconfiguration algorithm is iterative and during each iteration we search for multiple first-order branch exchanges that cumulatively offer a larger loss reduction compared to conventional single branch exchange based methods [32],[47]. A first-order branch exchange is achieved by closing 


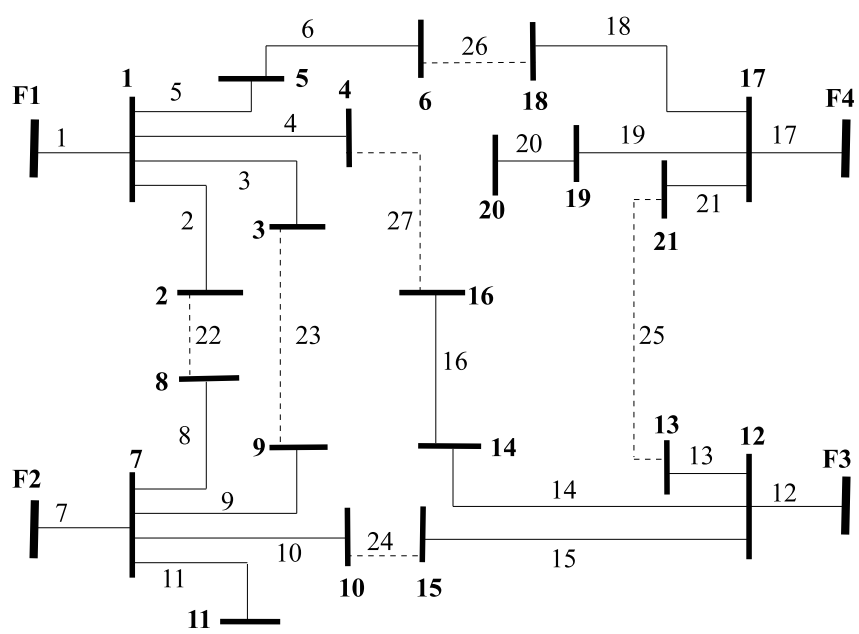

Fig. 3. Example of a power distribution network with four feeders. For simplicity the loads P,Q are not represented.

a tie switch and opening the closest sectionalizing switch. For example, in Fig. 3, the two first-order branch exchanges associated with switch 25 can be implemented by closing switch 25 and opening either switch 21 (load is transfered from feeder $F_{4}$ to feeder $F_{3}$ ) or switch 13 (load is transfered from $F_{3}$ to $F_{4}$ ). The key idea lies in the way we find the set of concurrent first-order branch exchanges. We do that by formulating this problem as a minimum cost maximum flow problem, whose solution will indicate the best set of branch exchanges for loss reduction. This formulation incurs the following two steps, which we will describe in the next sections:

- Construct the flow network $(F N)$ graph, $G(V, A)$, where $V$ and $A$ are the sets of nodes and arcs.

- Solve the MCMF problem.

1) Construction of the Flow Network Graph: The construction of the flow network graph $G(V, A)$ is crucial to the correctness of the proposed technique. To describe it easier, we use the example from Fig. 3 to illustrate the construction of the MCMF flow network graph presented in Fig. 4. The flow network graph is constructed using the following graph construction rules:

- The graph contains a source $s$ node and a sink $t$ node.

- The graph contains donor feeder, acceptor feeder, and switch nodes. There can be up to $2 F$ donor/acceptor feeder nodes and $S$ switch nodes, where $F$ is the number of feeders in the system and $S$ is the number of tie switches. A donor feeder node is associated with a feeder tree, which will transfer load (i.e., donate) to another feeder tree, associated with an acceptor node. A switch node $S_{u, v}$ represents the closing of tie switch $u$ and opening of sectionalizing switch $v$.

- A pair of arcs from a donor feeder node $F_{i}$ to an acceptor feeder node $F_{j}$ via a switch node $S_{u, v}$ is created only if a load transfer from feeder $F_{i}$ to feeder $F_{j}$ leads to a loss reduction. The loss reduction is used to compute the arc cost assigned to the first arc of the pair. Because we seek

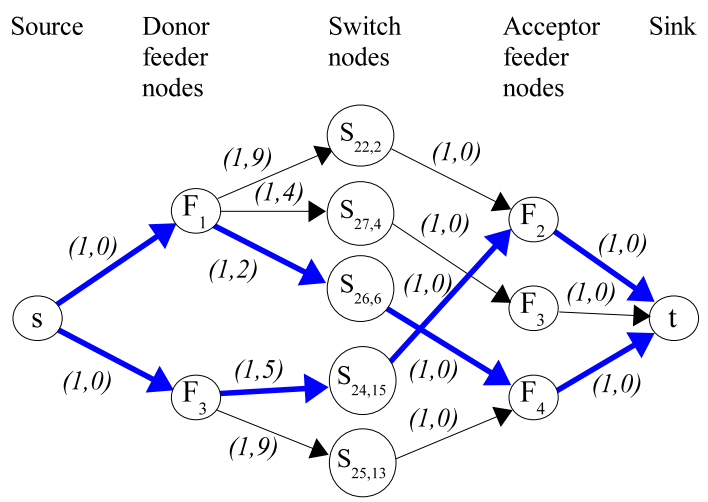

Fig. 4. Illustration of the flow network graph construction. Each arc is tagged with a (flow upper bound, cost) pair. The solution of the MCMF problem is highlighted using thicker arcs. A similar flow network is created during each iteration of the proposed MCMF based algorithm.

branch exchanges that lead to the largest loss reduction, the cost is inversely proportional to the loss reduction. All other arcs have a cost of zero and all arcs in the graph have a flow upper bound of 1 . For example, in Fig. 4, the pair of arcs between feeders $F_{1}$ and $F_{2}$ via $S_{22,2}$ represents a branch exchange. The loss reduction that would be achieved by this branch exchange is used to compute the cost of 9 assigned to arc $\left(F_{1}, S_{22,2}\right)$.

- If a pair of arcs is created between feeders $F_{i}$ and $F_{j}$ via switch $S_{u, v}$, then a pair of arcs between $F_{j}$ and $F_{i}$ through the same switch is prohibited. That is because loss reduction can be achieved in only one direction by closing a tie switch.

- If there are more possible branch exchanges between two feeders $F_{i}$ and $F_{j}$, only the one that leads to the maximum loss reduction is used in the graph construction. For example, in Fig. 3, even though there are two possible branch exchanges between feeders $F_{1}, F_{2}$ via switches 22 and 23 , only the branch exchange via switch 22 (due to its larger loss reduction) is included during the graph construction from Fig. 4.

2) Solving the MCMF problem: After the flow network graph is constructed using the procedure described in the previous section, the minimum cost maximum flow problem can be formally written, using the terminology from [57], as follows:

$$
\begin{aligned}
& \text { Minimize } \quad \sum_{(i, j) \in A} c_{i j} x_{i j} \\
& \text { Subject to } \sum_{j:(i, j) \in A} x_{i j}-\sum_{j:(j, i) \in A} x_{j i}=b(i) \quad \forall i \in V \\
& x_{i j} \in\{0,1\} \quad \forall(i, j) \in A
\end{aligned}
$$

where $x_{i j}$ is the flow through arc $(i, j) \in A$ and can be 0 or 1 because all arcs have unit capacity. $c_{i j}$ is the cost associated with each arc, and $b(i)$ is the supply (demand) associated with node $i \in V$.

We solve this MCMF problem using an efficient implementation of the relabel shortest path algorithm, 


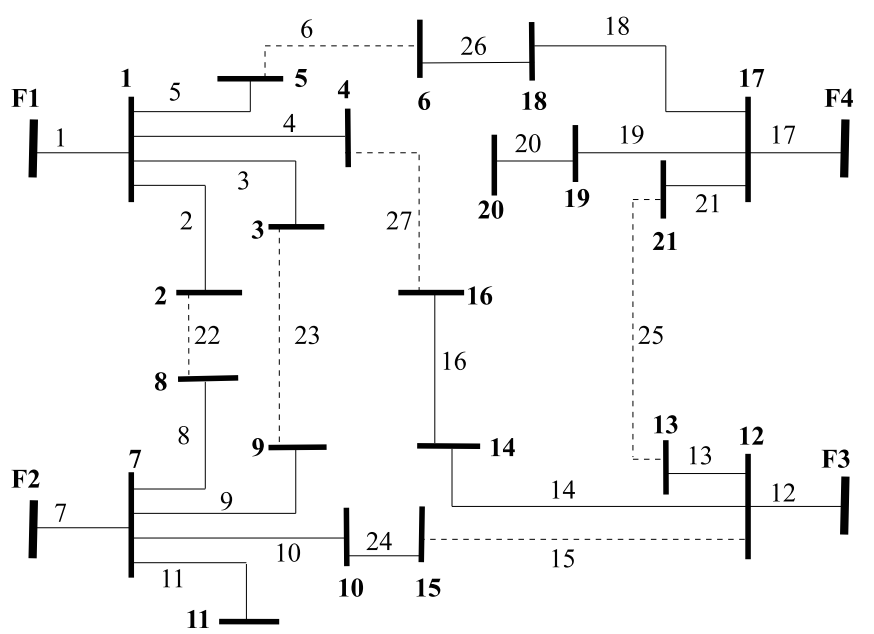

Fig. 5. Power distribution network from Fig. 3 after the implementation of the first-order branch exchanges indicated by the solution of the MCMF problem from Fig. 4.

which is described in detail in [57]. The solution of the MCMF problem practically dictates all the concurrent branch exchanges that we select to be implemented during the current iteration. In other words, the flows in the network flow graph found by the MCMF solution represent the load transfer between feeders, via the corresponding switches along the paths of the flows. For example, the solution of the problem in Fig. 4 is highlighted by using thicker lines that indicate concurrent load transfers between feeders $F_{1}, F_{4}$ and feeders $F_{3}, F_{2}$, which lead to maximum loss reduction. At the end of the current iteration, the power system is reconfigured by closing switches 26,24 and opening switches 15, 6. This new configuration (see Fig. 5) represents the starting configuration in the next iteration. We would like to emphasize that the number of concurrent branch exchanges dictated by the MCMF solution depends on the internal structure of the system (number of feeders) and the system size and can be any positive integer.

3) Loss Reduction During Each Iteration: During each iteration of the proposed reconfiguration algorithm, the solution of the MCMF problem indicates the best set of concurrent branch exchanges (that lead to maximum loss reduction during that iteration) to be performed. The cumulated loss reduction due to these branch exchanges is guaranteed to be maximized by the minimum cost maximum flow solution. This characteristic is unique to the proposed reconfiguration method and sets it apart from previous work. In this section, we use a simple example to show that sorting-based reconfiguration techniques would not be able to find the best solution, unless exhaustive solution enumeration is done, which would be prohibitively expensive. For example, let us consider in Table I the list of possible branch exchanges during one of the reconfiguration iterations. This is a distribution system example that has six feeders (substations) and 13 tie switches. For the sake of simplicity, its one line diagram is not presented in this paper. This list is derived using the general graph construction rules presented in the previous section.
If one were to select multiple branch exchanges by first sorting all switches by their associated loss reductions, and then selecting greedily as many independent branch exchanges (independent is defined as being between different feeders) as possible, then the solution $S_{4}, S_{13}, S_{5}$ (shown with bold fonts in the right hand-side of Table I) will be found. This solution has a cumulated loss reduction of 18 , which is not the best. The best solution is represented by $S_{9}, S_{13}, S_{3}$ that has a cumulated loss reduction of 21 . This solution is guaranteed to be found by the proposed in this paper technique due to the inherent MCMF problem formulation.

TABLE I

LIST OF LOSS REDUCTIONS ASSOCIATED WITH BRANCH EXCHANGES USED FOR CONSTRUCTING THE FLOW NETWORK GRAPH OF AN EXAMPLE DISTRIBUTION SYSTEM WITH SIX FEEDERS.

\begin{tabular}{cccccc}
\hline Switch & $\begin{array}{c}\text { Initial } \\
\text { Load transfer } \\
F_{i} \rightarrow F_{j}\end{array}$ & $\begin{array}{c}\text { Loss } \\
\text { reduction }\end{array}$ & Switch & $\begin{array}{c}\text { Load transfer } \\
F_{i} \rightarrow F_{j}\end{array}$ & $\begin{array}{c}\text { Loss } \\
\text { reduction }\end{array}$ \\
\hline \hline$S_{1}$ & $F_{1} \rightarrow F_{2}$ & 3 & $S_{4}$ & $F_{2} \rightarrow F_{4}$ & $\mathbf{9}$ \\
$S_{2}$ & $F_{1} \rightarrow F_{6}$ & 4 & $S_{9}$ & $F_{4} \rightarrow F_{1}$ & 9 \\
$S_{3}$ & $F_{2} \rightarrow F_{3}$ & 4 & $S_{12}$ & $F_{6} \rightarrow F_{4}$ & 8 \\
$S_{4}$ & $F_{2} \rightarrow F_{4}$ & 9 & $S_{13}$ & $F_{6} \rightarrow F_{5}$ & $\mathbf{8}$ \\
$S_{5}$ & $F_{3} \rightarrow F_{1}$ & 1 & $S_{7}$ & $F_{3} \rightarrow F_{5}$ & 6 \\
$S_{6}$ & $F_{3} \rightarrow F_{6}$ & 5 & $S_{6}$ & $F_{3} \rightarrow F_{6}$ & 5 \\
$S_{7}$ & $F_{3} \rightarrow F_{5}$ & 6 & $S_{2}$ & $F_{1} \rightarrow F_{6}$ & 4 \\
$S_{8}$ & $F_{4} \rightarrow F_{3}$ & 1 & $S_{3}$ & $F_{2} \rightarrow F_{3}$ & 4 \\
$S_{9}$ & $F_{4} \rightarrow F_{1}$ & 9 & $S_{1}$ & $F_{1} \rightarrow F_{2}$ & 3 \\
$S_{10}$ & $F_{4} \rightarrow F_{5}$ & 1 & $S_{5}$ & $F_{3} \rightarrow F_{1}$ & $\mathbf{1}$ \\
$S_{11}$ & $F_{5} \rightarrow F_{2}$ & 1 & $S_{8}$ & $F_{4} \rightarrow F_{3}$ & 1 \\
$S_{12}$ & $F_{6} \rightarrow F_{4}$ & 8 & $S_{10}$ & $F_{4} \rightarrow F_{5}$ & 1 \\
$S_{13}$ & $F_{6} \rightarrow F_{5}$ & 8 & $S_{11}$ & $F_{5} \rightarrow F_{2}$ & 1 \\
\hline
\end{tabular}

\section{EXPERIMENTAL RESULTS}

The proposed algorithm was implemented in $\mathrm{C}++$ and simulations were performed on a Linux machine running on a $2.8 \mathrm{GHz}$ Intel Quad processor with $2 \mathrm{~GB}$ memory. We report the loss reduction achieved with the proposed MCMF based algorithm versus the traditional Baran's method on several power systems with sizes ranging from 83 to 10476 buses. The results are presented in Table II. The first power system testcase is from [8], the next two testcases are from [7], and the last two testcases are artificially created using data from the cited testcases.

As shown in Table II, the solution achieved using the proposed MCMF based algorithm is similar to that achieved using the Baran's method (i.e., with similar losses) for testcases with smaller size, but with significantly fewer iterations. This is because the flow network solution identifies multiple branch exchanges that yield larger loss reductions in each iteration, which in turn leads to faster convergence. As the testcase size increases, the proposed reconfiguration algorithm improves the solution quality significantly. This can be explained by the fact that the MCMF solution is able to identify the best concurrent branch exchanges during each iteration, especially when the number of possible branch exchanges increases. The runtime of both reconfiguration algorithms is governed by the number of times the power flow is executed. Because the proposed algorithm terminates in much fewer iterations, the runtime savings become significant, leading to an average of $2.3 \times$ speedup. It is to be noted that the proposed algorithm additionally 
TABLE II

NETWORK FLOW BASED VERSUS BARAN'S RECONFIGURATION ALGORITHM [32]. THE POWER FLOW SOLUTION IS COMPUTED USING DISTFLOW [56] FOR BOTH RECONFIGURATION ALGORITHMS

\begin{tabular}{lccccccc}
\hline & \multicolumn{2}{c}{ Baran's reconfiguration [32] } & \multicolumn{2}{c}{ Proposed reconfiguration } & Speed-up \\
\hline Testcase & Loss red. \% & Iter. Num. & CPU [ms] & Loss red. \% & Iter. Num. & CPU [ms] \\
\hline \hline bus_83_11 & 12.36 & 11 & 13.3 & 12.36 & 5 & 7.8 & 1.71 \\
\hline bus_135_8 & 13.54 & 17 & 17.1 & 13.54 & 8 & 9.8 & 1.74 \\
\hline bus_201_3 & 6.74 & 22 & 30.6 & 6.74 & 13 & 17.7 & 1.73 \\
\hline bus_873_7 & 69.09 & 104 & 476.4 & 69.36 & 57 & 271.3 & 1.76 \\
\hline bus_10476_84 & 36.98 & 275 & 14711.1 & 47.58 & 56 & 3432.3 & 4.55 \\
\hline \multicolumn{1}{r}{} \\
\hline
\end{tabular}

requires the runtime overhead responsible for constructing and solving the network-flow problem. This runtime overhead (included in the results reported in Table II) is negligible for all testcases especially because the size of the network-flow graph is small. That is, the MCMF problem size (as number of vertices of the network-flow graph) is bound by $2 F+S$, where $F$ is the number of feeders and $S$ is the number of tie switches in the system.

\section{A. Discussion}

In order to better illustrate the behavior of the proposed algorithm, we plot in Fig. 6 the percentage of loss reduction achieved during each iteration of the reconfiguration algorithms for the first testcase bus_83_11. It can be seen that, for example, the proposed network-flow based reconfiguration algorithm reduces losses with $7.85 \%$ and $3.35 \%$ during the first and second iterations, out of a total of $12.36 \%$ during a total of five iterations. The same amount of loss reduction is achieved only after five iterations using Baran's method, out of a total of eleven iterations. Similar behavior was also observed for the other distribution systems. Table III reports the number of first iterations required by each of the two reconfiguartion algorithms in order to achieve at least $95 \%$ of the final loss reduction. For example, the proposed reconfiguration algorithm achieves $98 \%$ of the total loss reduction of 12.36 during the first two iterations while the Baran's method needs five iterations to achieve $99 \%$ of the same total of 12.36 loss reduction for the first distribution system bus_83_11.

The solution quality achieved using the proposed algorithm is similar or better than that achieved using the traditional Baran's method and with significantly fewer iterations. It is important to note that even a small percentage reduction in losses can translate into substantial cost savings. For example, a $0.5 \%$ (from $3.5 \%$ to $3.0 \%$ ) reduction in losses equates to savings of $\$ 50$ million per year for the state of California [1].

Finally, in order to compare the runtime of the proposed algorithm with other previous approaches, we list in Table IV previously reported results and the corresponding system sizes in terms of number of buses. This way, we attempt only a qualitative comparison, because the runtimes reported in this paper and in previous work depend on the differences in processor speeds, memory used, and algorithm implementation. We note that the majority of previous work does not report details on computational runtimes. Moreover, algorithm implementations are not publicly available for comparison purposes. It can be observed that the proposed algorithm is one

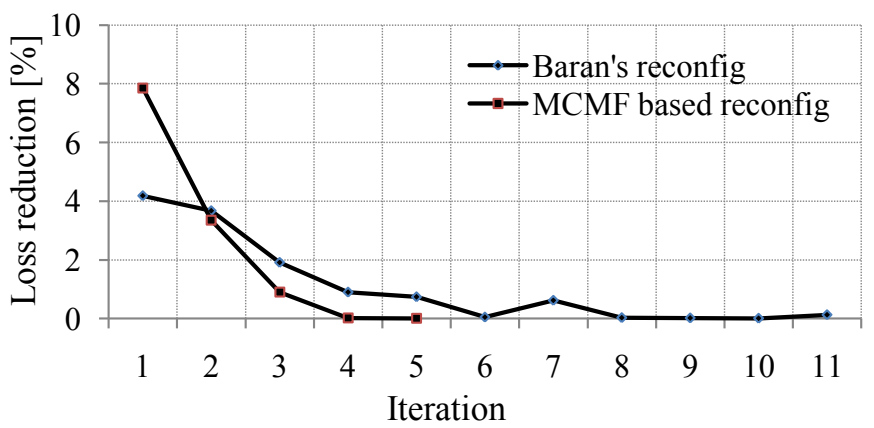

Fig. 6. Percentage of loss reduction achieved during iterations of the proposed algorithm and of Baran's reconfiguration algorithm for the first testcase distribution system bus_83_11.

TABLE III

NUMBER OF ITERATIONS REQUIRED TO ACHIEVE AT LEAST 95\% OF THE TOTAL LOSS REDUCTION.

\begin{tabular}{lcccc}
\hline & \multicolumn{2}{c}{ Baran's reconfiguration [32] } & \multicolumn{2}{c}{ Proposed reconfiguration } \\
\hline \multirow{2}{*}{ Testcase } & $\begin{array}{c}\text { Fraction of total } \\
\text { loss reduction } \%\end{array}$ & $\begin{array}{c}\text { Iter. } \\
\text { Num. }\end{array}$ & $\begin{array}{c}\text { Fraction of total } \\
\text { loss reduction } \%\end{array}$ & $\begin{array}{c}\text { Iter. } \\
\text { Num. }\end{array}$ \\
\hline \hline bus_83_11 & 99 & 5 & 98 & 2 \\
\hline bus_135_8 & 96 & 11 & 95 & 6 \\
\hline bus_201_3 & 95 & 14 & 97 & 8 \\
\hline bus_873_7 & 95 & 49 & 95 & 23 \\
\hline bus_10476_84 & 95 & 154 & 95 & 21 \\
\hline
\end{tabular}

of the fastest and therefore can be used as an efficient solution for online distribution system reconfiguration with application to distribution automation.

\section{CONCLUSION}

In this paper, we proposed a novel and efficient iterative heuristic algorithm for solving the network reconfiguration problem for loss reduction. The novelty of our approach lies in the network-flow based branch exchanges technique that leads to maximum loss reductions and faster convergence. Experimental results using this new approach on several power systems with sizes ranging from 83 to 10476 buses demonstrated that the new algorithm achieves similar or better loss reduction compared to previous work, but with significantly shorter runtimes. Therefore, the proposed algorithm represents an appealing solution to addressing the need for faster simulation and modeling with application to distribution automation. We are currently working on extending the proposed reconfiguration algorithm to also address meshed distributions systems. 
TABLE IV

QUALITATIVE COMPARISON OF COMPUTATIONAL RUNTIMES OF THE PROPOSED MCMF BASED RECONFIGURATION ALGORITHM WITH PREVIOUS APPROACHES

\begin{tabular}{lccc}
\hline Approach & CPU & System size & Processor \\
& runtime [s] & Num. buses & Memory \\
\hline \hline Proposed MCMF & 0.017 & 201 & $2.8 \mathrm{GHz}$ Intel Quad, 2 GB \\
& 0.271 & 873 & \\
& 3.43 & 10476 & \\
\hline Baran's reconfiguration [32] & 0.030 & 201 & $2.8 \mathrm{GHz}$ Intel Quad, 2 GB \\
& 0.476 & 873 & \\
\hline Tabu search [7] & 14.71 & 10476 & \\
\hline Ant colony [8] & 46 & 135 & $2.4 \mathrm{GHz}$ AMD Athlon, 512 MB \\
\hline Genetic algorithm [8] & 49 & 202 & NA \\
\hline Simulated annealing [8] & 241 & 96 & NA \\
\hline Multi-tier heuristic [34] & 303 & 96 & NA \\
\hline Sensitivity heuristic [48] & 257 & 96 & $200 \mathrm{MHz}$ Pentium, 32 MB \\
\hline
\end{tabular}

\section{REFERENCES}

[1] S. Price et al., "Preliminary Assessment: Value of Distribution Automation Applications," California Energy Commission, PIER, Publication No. CEC 500-2007-028, 2006, [Online]. Available: http://www.energy.ca.gov/2007publications/CEC-500-2007-028/CEC500-2007-028.PDF

[2] D.V. Dollen, "Enabling Energy Efficiency IntelliGrid," 2006 NARUC Summer Meeting, 2006, [Online]. Available: http://mydocs.epri.com/docs/CorporateDocuments/AssessmentBriefs/ NARUC_Intelligrid.pdf

[3] K. Nara, A. Shiose, M. Kitagawoa, T. Ishihara, "Implementation of genetic algorithm for distribution systems loss minimum reconfiguration," IEEE Trans. Power Systems, vol. 7, pp. 1044-1051, 1992.

[4] M. Kitayama, K. Matsumoto, "An optimization method for distribution system configuration based on genetic algorithm," Int. Conf. on Advances in Power System Control, Operation and Management (APSCOM), pp. 614-619, 1995.

[5] W.M. Lim, F.-S Cheng, M.-T. Tsay, "Distribution feeder reconfiguration with refined genetic algorithm," IEEE Proc. Power Generation Transmisison and Distribution, vol. 146, no. 6, pp. 349-354, Nov. 2000.

[6] Y. Mishima, K. Nara, T. Satoh, T. Ito, H. Kaneda, "Method for minimum-loss reconfiguration of distribution system by tabu search," Electrical Eng. Jpn., vol. 152, no. 2, pp. 18-25, 2005.

[7] M.A.N. Guimaraes, C.A. Castro, "Reconfiguration of Distribution Systems for Loss Reduction Using Tabu Search," IEEE Power System Computation Conference (PSCC), vol. 1, pp. 1-6, Aug. 2005.

[8] C.-T. Su, C.-F. Chang, J.-P. Chiou, "Distribution network reconfiguration for loss reduction by ant colony search algorithm," Electric Power Systems Research, vol. 75, no. 23, pp. 190-199, Aug. 2005.

[9] H.C. Chang, C.C. Kuo, "Network reconfiguration in distribution system using simulated annealing," Elect. Power Syst. Res., vol. 29, pp. 227238, 1994.

[10] V. Parada, J.A. Ferland, M. Arias, K. Daniels, "Optimization of electrical distribution feeders using simulated annealing," IEEE Trans. Power Delivery, vol. 19, no. 3, pp. 1135-1141, July 2004.

[11] H. Kim, Y.K. Ko, H. Jung, "Artificial neural networks based feeder reconfiguration for loss reduction in distribution systems," IEEE Trans. Power Delivery, vol. 8, pp. 1356-1366, July 1993.

[12] J. Olamaei, G. Gharehpetian, T. Niknam, "An approach based on particle swarm optimization for distribution feeder reconfiguration considering distributed generators," Power Systems Conference (PSC), pp. 326-330, Mar. 2007.

[13] W.W. Chang, M.-S. Tasi, F.-Y. Hsu, "A new binary particle swarm optimization for feeder reconfiguration," Intelligent Systems Applications for Power Systems (ISAP), pp. 1-6, Nov. 2007.

[14] K. Prasad, R. Ranjan, N.C. Sahoo, A. Chaturvedi, "Optimal reconfiguration of radial distribution systems using a fuzzy mutated genetic algorithm," IEEE Trans. Power Delivery, vol. 20, no. 2, pp. 1211-1213, Apr. 2005.

[15] D. Das, "A fuzzy multiobjective approach for network reconfiguration of distribution systems," IEEE Trans. Power Delivery, vol. 21, no. 1, pp. 202-209, Jan. 2006.

[16] Y.H. Song, G.S. Wang, A.T. Johns, P.Y. Wang, "Distribution network reconfiguration for loss reduction using fuzzy controlled evolutionary programming," IEE Gen. Transm. Dist., vol. 144, no. 4, pp. 345-350, July 1997.

[17] Y.-T. Hsiao, "Multiobjective Evolution Programming Method for Feeder Reconfiguration," IEEE Trans. Power Systems, vol. 19, no. 1, pp. 594-598, Feb. 2004

[18] H.D. Chiang, J.J. Rene, "Optimal network reconfiguration in distribution systems: Part 1: A new formulation and a solution methodology," IEEE Trans. Power Delivery, vol. 5, no. 4, pp. 1902-1908, Oct. 1990.

[19] H.D. Chiang, J.J. Rene, "Optimal network reconfiguration in distribution systems: Part 2: Solution algorithms and numerical results," IEEE Trans. Power Delivery, vol. 5, no. 3, pp. 1568-1574, Jul. 1992.

[20] C.-T. Su, C.-S. Lee, "Feeder reconfiguration and capacitor setting for loss reduction of distribution systems," Electric Power Systems Research, vol. 58, no. 2, pp. 97-102, June 2001.

[21] E.M. Carreno, R. Romero, A. Padilha-Feltrin, "An Efficient Codification to Solve Distribution Network Reconfiguration for Loss Reduction Problem," IEEE Trans. Power Systems, vol. 23, no. 4, pp. 1542-1551, Nov. 2008.

[22] D. Zhang, Z. Fu, L. Zhang, "Joint Optimization for Power Loss Reduction in Distribution Systems," IEEE Trans. Power Delivery, vol. 23, no. 1, pp. 161-169, Feb. 2008.

[23] C.C. Liu, S.J. Lee, S.S. Venkata, "An expert system operational aid for restoration and loss reduction of distribution system," IEEE Trans. Power Systems, vol. 3, pp. 619629, May 1988.

[24] T.P. Wagner, A.Y. Chikani, R. Hackam, "Multi-objective feeder reconfiguration by distribution management systems", IEEE Trans. Power Systems, vol. 11, no. 2, pp. 661-667, May 1996.

[25] A.C.B. Delbem, N.G. Bretas, A. De Carvalho, "Distribution system reconfiguration using graph chain representation," Int. Conf. on Power System Technology, vol. 1, pp. 439-443, 2000.

[26] A.C.B. Delbem, A.C.Pd.L.F. de Carvalho, N.G. Bretas, "Main chain representation for evolutionary algorithms applied to distribution system reconfiguration," IEEE Trans. Power Systems, vol. 20, no. 1, pp. 425-436, Feb. 2005 .

[27] J. Olamaei, T. Niknam, G. Gharehpetian, "Impact of Distributed Generators on Distribution Feeder Reconfiguration," IEEE Power Tech, vol. 1, no. 1-5, pp. 1747-1751, July 2007.

[28] T.P. Wagner, A.Y. Chikhani, R. Hackam, "Feeder reconfiguration for loss reduction: an application of distribution automation," IEEE Trans. Power Delivery, vol. 6, no. 4, pp. 1922-1933, Oct. 1991.

[29] R.J. Sarfi, M.M.A. Salama, A.Y. Chikhani, "A survey of the state of the art in distribution system reconfiguration for system loss reduction" Electric Power Systems Research, vol. 31, pp. 61-70, 1994.

[30] N.G. Caicedo, C.A. Lozano, J.F. Diaz, C. Rueda, G. Gutierrez, C. Olarte, "Loss reduction in distribution networks using concurrent constraint programming" Probabilistic Methods Applied to Power Systems, pp. 295-300, Sep. 2004.

[31] S. Civanlar, J.J. Grainger, H. Yin, S.S.H. Lee, "Distribution feeder reconfiguration for loss reduction," IEEE Trans. Power Delivery, vol. 3, no. 3, pp. 1217-1223, July 1988.

[32] M.E. Baran, F.F. Wu, "Network reconfiguration in distribution systems for loss reductionand load balancing," IEEE Trans. Power Delivery, vol. 4, no. 2, pp. 1401-140, Apr. 1989.

[33] M.A. Kashem, G.B. Jasmon, V. Ganapathy, "A new approach of distribution system reconfiguration for loss minimization," Int. J. of 
Electrical Power and Energy Systems, vol. 22, no. 4, pp. 269-276, May 2000.

[34] K.N. Miu, H.-D. Chiang, R.J. McNulty, "Multi-tier service restoration through network reconfiguration and capacitor control for large-scale radial distribution networks," IEEE Trans. Power Systems, vol. 15, no. 3, pp. 1001-1007, Aug. 2000.

[35] C.-F. Chang, "Reconfiguration and Capacitor Placement for Loss Reduction of Distribution Systems by Ant Colony Search Algorithm," IEEE Trans. Power Systems, vol. 23, no. 4, pp. 1747-1755, Nov. 2008.

[36] U. Eminoglu, M.H. Hocaoglu, "A new power flow method for radial distribution systems including voltage dependent load models," Electric Power Systems Research, vol. 76, no. 1-3, pp. 106-114, Sep. 2005.

[37] R.J. Sarfi, M.M.A. Salama, A.Y. Chikhani, "Distribution system reconfiguration for loss reduction: analgorithm based on network partitioning theory," IEEE Trans. Power Systems, vol. 11, no. 1, pp. 504-510, 1996.

[38] J.-C. Wang, H.-D. Chiang, G.R. Darling, "An efficient algorithm for real-time network reconfiguration inlarge scale unbalanced distribution systems," IEEE Trans. Power Systems, vol. 11, no. 1, pp. 511-517, 1996.

[39] D. Shirmohammadi, H.W. Hong, "Reconfiguration of electric distribution networks for resistive line loss reduction," IEEE Trans. Power Delivery, vol. 4, no. 2, pp. 1492-1498, Apr. 1989.

[40] V. Borozan, D. Rajicic, R. Ackovski, "Minimum loss reconfiguration of unbalanced distribution networks," IEEE Trans. Power Delivery, vol. 12, pp. 435-441, Jan. 1997.

[41] C.C. Liu, S.J. Lee, K. Vu, "Loss minimization of distribution feeders: optimality and algorithms," IEEE Trans. Power Delivery, vol. 4, pp. 1281-1289, Apr. 1989.

[42] S.K. Goswani, S.K. Basu, "A new algorithm for the reconfiguration of distribution feeders for loss minimization," IEEE Trans. Power Delivery, vol. 7, pp. 78-88, Jan. 1991.

[43] J.S. Wu, K.L. Tomsovic, C.S. Chen, "A heuristic search approach to feeder switching operations for overload, faults, unbalanced flow and maintenance," IEEE Trans. Power Delivery, vol. 6, pp. 1579-1585, Oct. 1991.

[44] T. Taylor, D. Lubkeman, "Implementation of heuristic search strategies for distribution feeder reconfiguration," IEEE Trans. Power Delivery, vol. 5, no. 1, pp. 239-246, Jan 1990.

[45] H. Shaibon, S. Murni, B. Susyeto, A.A.M. Zin, K.L. Lo, "Loss reduction in power distribution by means of switching factors method," Intl. Conf. Advances in Power Systems Control Operation and Management (APSCOM), vol. 1, pp. 203-208, Nov. 1997.

[46] V.C. Veera Reddy, N. Perumal, Y. Rajasekharaeddy, "A Loss reduction in distribution networks: a two stage solution approach," Proc. National Power and Energy Conference (PECon), pp. 241-246, Nov. 2004.

[47] G.K.V. Raju, P.R. Bijwe, "An Efficient Algorithm for Loss Reconfiguration of Distribution System Based on Sensitivity and Heuristics," IEEE Trans. Power Systems, vol. 23, no. 3, pp. 1280-1287, Aug. 2008.

[48] F.V. Gomes, J.R. Carneiro, J.L.R. Pereira, "A New Distribution System Reconfiguration Approach Using Optimal Power Flow and Sensitivity Analysis for Loss Reduction," IEEE Trans. Power Systems, vol. 21, no. 4, pp. 1616-1623, Nov. 2006.

[49] H.M. Khodr, J.M. Crespo, M.A. Matos, J. Pereira, "Distribution Systems Reconfiguration Based on OPF Using Benders Decomposition," IEEE Trans. Power Delivery, vol. 24, no. 4, pp. 2166-2176, Oct. 2009. Autor/es:

[50] H.P. Schmidt, N. Kagan, "Fast reconfiguration of distribution systems considering loss minimization," IEEE Trans. Power Systems, vol. 20, no. 3, pp. 13111319 , Aug. 2005.

[51] D.-J. Shina, J.-O. Kima, T.-K. Kimb, J.-B. Choob, C. Singh, "Optimal Service Restoration and Reconfiguration of Network Using GeneticTabu Algorithm," Electric Power Systems Research, vol. 71, no. 2, pp. 145-162, Oct. 2004.

[52] A. Ahuja, S. Das, A. Pahwa, "An AIS-ACO Hybrid Approach for Multi-Objective Distribution System Reconfiguration," IEEE Trans. Power Systems, vol. 22, no. 3, pp. 1101-1111, Aug. 2007.

[53] J.P. Chiou, C.-F. Chang, C.-T. Su, "Variable scaling hybrid differential evolution for solving network reconfiguration of distribution systems," IEEE Trans. Power Systems, vol. 20, no. 2, pp. 668674, May 2005.

[54] C.T. Su, C.S. Lee, "Network reconfiguration of distribution systems using improved mixed-integer hybrid differential evolution," IEEE Trans. Power Delivery, vol. 18, no. 3, pp. 1022-1027, Jul. 2003.

[55] D.E. Bouchard, A.Y. Chikani, V.I. John, M.M.A. Salama, "Applications of Hopfield neural networks to distribution feeder reconfiguration," Intl. Forum Applications of Neural Networks to Power Systems (ANNPS), pp. 311-316, 1993
[56] M.E. Baran, F.F. Wu, "Optimal sizing of capacitors placed on a radial distribution system," IEEE Trans. Power Delivery, vol. 4, no. 1, pp. 735-743, Jan. 1989.

[57] R.K. Ahuja, T.L. Magnanti, J.B. Orlin, Network Flows: Theory, Algorithms, and Applications. Prentice Hall, 1993. 